\title{
Individual Building Growth Model Based on Column Grid Cellular Automata
}

\author{
Gongyu Hou and $\mathrm{Xin} \mathrm{Xu}^{*}$
}

\author{
School of Mechanics and Civil Engineering, China University of Mining \& Technology (Beijing), 100083, P.R. China
}

\begin{abstract}
Architectural generative design has gained an overwhelming contemporary popularity when it comes to the digital architecture research. This paper proposes a model which mimics the growth of plants. Take the growth of a plant for example, branches grow before leaves. Similarly, the inward streamlines initiated from various entrances and exits within the boundaries of individual building are like branches, so are the functional areas to leaves. With the help of this idea, a growth model framework based on flat column grid cellular automata has been established which develops organic integration between CA model, site column grid and spatial database model. This model not only simulates the expansion of architectural space, but also reflects the building spatial variation of internal structure. It is a space-time dynamic model with basic features of complex systems and has great practical reference value for architects to understand the evolution process of architectural space. To help designers improve the efficiency of project decision, this paper uses genetic algorithm to obtain the positioning of function area, so as to effectively simulate the thought process of a successful architect.
\end{abstract}

Keywords: Cellular automata, growth model, individual building.

\section{INTRODUCTION}

Cellular automata (Cellular Automaton), referred to as CA, has been widely applied in social, economic, military and scientific fields ever since its existence, as it can be used to study various common phenomena, such as communication, messaging, calculation, structure growth [1], replication, competition and evolution. As a discrete dynamical system regarding both time and space, spreading each regular grid Cellular (Cell) takes finite discrete state, followed by the same rules of action and is determined in accordance with the local rules for synchronous update, since a large number of simple cellular interactions constitute the evolution of dynamic systems. Its dynamic behavior can be categorized into four types:

(1) Smooth type: from any initial state, after a certain period of running, every cellular remains in a fixed state and does not change with time.

(2) Cyclical type: after a certain time of operation, cellular space tends to have a series of simple periodic structure.

(3) Chaos type: after a certain period of running, cellular automata exhibits chaotic aperiodic behavior and manifests itself in fractal dimension features.

(4) Complex type: partial chaos, some of which will continue to spread.

In geographical [2], urban [3], and architectural [4] studies, CA can be simulated on the temporal and spatial variation. In terms of simulation object, CA simulation requires

*Address correspondence to this author at the School of Mechanics and Civil Engineering, China University of Mining \& Technology (Beijing), Xueyuan road, Haidian District, Beijing, 100083, P.R. China;

Tel: +86010 13466594540; E-mail: boshimai@yeah.net different resolution raging from earth to rooms inside a building. Individual building can be divided into functional areas, whereas functional areas are consisted of different rooms. On the other hand, individual building can be seen as a "cellular" after division, which is the scope of site analysis. If a site is a construction project, it can be seen as a "cellular" of urban functional area, which is the scope of urbanology. Furthermore, urban can be seen as a "cellular" of functional area in certain regions, so are regions to the country as a whole on a larger scale, which is the scope of geographical analysis. In summary, all these resolution objects can be used to simulate the evolution process dynamically using CA.

\section{CONCEPT OF COLUMN GRID CELLULAR AU- TOMATA}

The way this article explores 'Cellular is unique', which is proposed for building growth models [5]. In a venue such as a factory or residential area, whenever it requires several construction phases, it involves scheme design of individual buildings. Scheme design can be further divided into two stages, including design of functional areas and arrangement design of rooms within the functional zones [6]. As is shown in Fig. (1), on the basis of same functionality bubble condition, due to differences in design ideas, it results in a four function modeling [7]. Connection points between functional areas include two cases: one is a corridor, the other is connected to each other with at least one column spacing. If functional areas are connected with corridors, then they will look like "octopus", under which condition there would be at least hundreds of function modeling that meet the functional condition of the bubble function modeling plans. In fact, the merits of a design can be determined at the functional modeling stage. The next stage is to arrange rooms within the func- 
tional districts, and the main task of this phase is to specify the traffic line position in the functional area, and then combine the rooms together using streamlines.

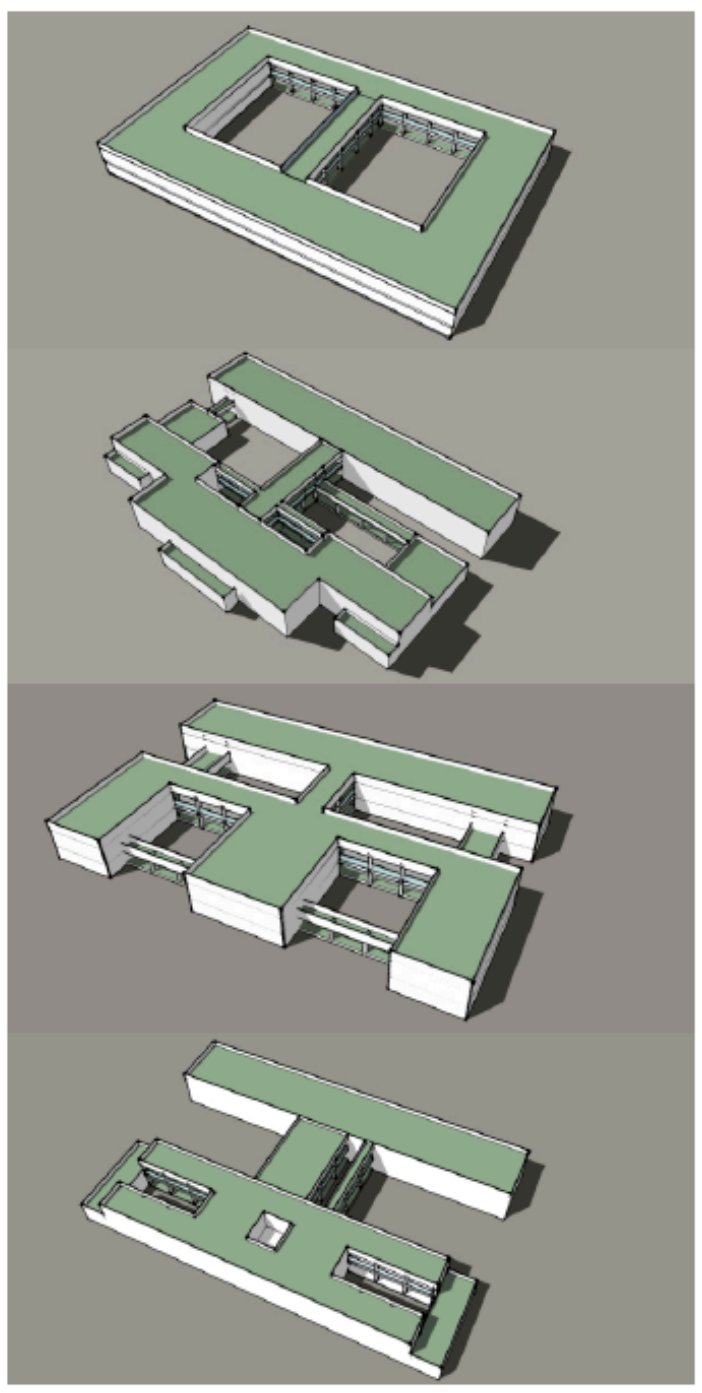

Fig. (1). Four different functional modeling SketchUp.

Currently, most buildings are built using frame structure. As a result, the cellular grid can be seen as regular column grid, which is a discrete architecture space and each cellular is a space surrounded by four frame columns.

For individual building generation, the proposed model mimics the growth of plants. From within the boundaries of different building entrances, inward birth transit transport "energy" --- personnel and material (flow lines), transit thickness of a column spacing, as plant grows before long branches, and then long leaves, this transit is a branch, and functional area is the leaves. As a matter of fact, this transit is a major transportation channel within a single building, and is also produced according to the conditions generated by the bubble chart. It is a criteria that must be met, which does not explicitly requires the evacuation of transit in the function bubble chart. Otherwise under an implied condition, if mathematical descriptions were generated, the evacuation distance conditions must be listed.
Considering the growth of entrances and exit conditions will simplify functional areas, access is limited to conditions of each functional area. Except for cellular number, boundaries and function code restrictions, conditions of flow lines are key conditions, which essentially are entrances or Outreach conditions. Each functional area has at least one external connection port, and has three external connection ports on average, some of which are evacuation routes, and are closed under normal conditions. So, the streamline simulation should consist of both normal flow line and emergency flow line, where the evacuation channel is enabled only if emergencies happen. Normally, each functional area is set up with at least one vertical transportation space, which is shown in the form of stairs, elevators, escalators and ramps.

Functional modeling phase is essentially the aggression of functional area cellular in the building boundaries, which then moves according to the conditions of outreach, accompanied by mutual position adjustment. The adjustment process must meet the lighting conditions, movement, and other internal and external conditions, which are given implicitly in the outreach conditions. At room arrangement design stage, cellular level degrade from the size of the column to grid $300 \mathrm{~mm}$ size, whereas columns, room and window size are generally multiples of the basic modulus. One column can be refined as the cellular network of hundreds of basic cellular, whose exact size fits human scale precisely, and therefore it can use human agent simulation for construction.

There is no doubt that branches of traffic lines [8] are the central region of functional areas which means they are the most important areas, and it is similar to the reason why urban cities appear prosperous in traffic arteries intersections (land road and waterway) geographically. So the growth of functional areas and transit meet fractal growth characteristics, while the fractal is the geometric description language of the space we live in. Transform flat transit lines grow into program modules, and they can be used in different resolutions of the growth environment etc. [9-11].

This paper discusses the growing functional modeling area, which follows several principles:

(1) Central principle: the center is the intersection of the transit lines, which manifest as hall inside the building, the active area around the center, and the focus of the functional areas should be put within the hall in program calculation.

(2) Symmetry principle: Ribbon shape symmetry, or axis of symmetry, or center of symmetry.

(3) Lighting principles: a relatively short ribbon edge with no more than 3 column spacing.

(4) Collision detection principle: each step should be carried out to detect whether they encounter other functional areas.

(5) Coding principles: generate functional areas according to the coding sequence.

\section{FLAT RIBBON GROWTH MODEL FRAMEWORK}

The growth model consists of three parts, namely three levels. The first part is the ribbon type level; the second part is the flow line level; the third part is the control influential 
factor level under upper ribbon resolution. They combine with each other through cellular structure of column grid level resolution.

The first type is the core part of the functional areas of the model, which is based on a CA model of different functional areas, not including cellular flow line. Interactions and dynamics of the different functional areas of the building resulted in dynamic growth [12] modeling. When the functional area unit determines its behaviors on the basis of adjacent functional area structures, its rules of conduct are also affected by the next two levels at the same time.

The second portion is shunt line level and has double characteristics: on the one hand, by its reachability index obtained as the above-mentioned factors and control factors of the functional units change, it controls cellular behavior; on the other hand, it can also be a dynamic CA model and simulate evolving transportation network, then functional areas serve as factors affecting the flow lines and control layer. Therefore, the growth models are coupled complex interactions CA model of the streamline and functional models.

The third part of the factors affecting the level of control is the level of GIS spatial regions with different characteristics. As external growth model building environment, it influences and controls the behavior of the above functions and streamline units. Influential factors include the regulatory detailed planning of venue, landscaping, water, noise and so on. In consideration of model performance and other aspects of the model utilities, there is only one GIS integrated control layer. The control layer has two forms: one can be used for the results of spatial analysis using spatial duplication of several control factors level, when the control level reflects the suitability of buildings and is a static level. Another form can be a dynamic prediction model based on the venue. CA model of the above two parts operates synchronously and interacts with each of the other architectural planning indicators deriving from building organic growth model based the venue model. And, the correlation between these indicators function and flow lines, adjusts the rules of conduct parameters of column grid CA model, whether to restrict or promote the growth of certain types of functions, or control operation of the site column grid CA model from the aspect of venue level.

Through there are three parts under this unified framework, building organic growth model will be integrated with the site model and spatial database column grid CA model, making the building organic growth model more than just a column grid CA model, but as an integrated model of column grid venue, which greatly improves the usefulness of the model.

\section{BUILDING FLAT RIBBON GROWTH MODEL CHARACTERISTICS}

In this paper, building growth models extend the traditional cellular automata model in many ways.

First, the construction growth model is an object-oriented cellular automaton model. In the traditional model of cellular automata, cellular state has only one discrete value, which is apparently unable to meet the needs of architectural space.
Section III discusses the expansion theory of extend cellular from a single state variable space to physical objects in space, in the model structure, the use of the concept of agent, sort functional areas and flow lines into different space entity classes, giving it different state variables and rules of conduct.

Second, the construction growth model is a stochastic cellular automata model. In the model, the transition rules are controlled by the probability function, which are random processes based on probability and make extensive uses of Monte Carlo methods. By using a series of random numbers to express a probability distribution, in essence, of a class through the relevant random variables or random sampling process to solve complex random phenomena. The basic idea is to give the quantitative description of complex stochastic systems so as to solve certain mathematical problems identified, which requires to artificially construct a system that corresponds to the original, but is not directly related to the probability of the process system, using a large number of random statistical phenomenon brought by this system to describe, and simulate the real system behavior and the law.

Third, the growth model is a different building configurations cellular automaton model. Different configurations of cellular automata models are those whose rules change over time or space. For construction growth model, on the one hand, due to the introduction of the control factor model as the external environment of cellular changes, rules of conduct of each cell varies because of their different external environment. On the other hand, construction growth model uses an object-oriented approach for cellular classified package. Therefore, not only different cellular have different cellular neighbors and conversion rules, but also as a space unit, it may perform different development rules at different development stage due to different production time. From this aspect, the growth model is a different building configurations cellular automaton model, which has far-reaching meaning for generating real buildings.

Fourth, the building growth model is still a peculiar linkage of two special CA models. Section III discusses the model framework, the functional layer and the layer flow lines are a cellular automaton model, but the two are not independent, under unified spatial reference system, functional and streamline layers interact and interrelate with each other, constituting a particular compound of this building growth model.

Fifth, the building growth model is a model for adaptive computing. Construction growth model calculates the appropriate initial column spacing according to model adaptive input of room size. Column grid form a cellular automata grid within the building line, whose outer boundary of the initial cellular grids is set up by the building line. According to the initial approximate location of the entrance at the outer boundary of the flow line given by the streamline level, functional area in column formation generates online adaptive sideline single architecture. According to the summary of the model functional room area, the number of cellular adaptation is used to calculate the various functional areas, and describe the function of the bubble chart on the cellular level functions performed. Module calculates the cellular amount of each functional area regarding the functional area 
size of rooms, whereas description of functional bubble chart is conducted at the level of functional cellular layer.

Sixth, site control cellular levels constitute the urban and rural growth space model. Venue resembles soil and growth environment of plants, which provides water, fertilizer, sunshine, growing space. Road construction near the boundaries is alike the nutrients around the roots of plants and seeds are sowed at the intersection of the ribbon streamlines. Roots will extend to the entrances and exits in the adjacent surroundings and various entrances and exits connect different functional areas, which itself is a grown tree. Entire single building is a small wood, the city's buildings communities are an analogy to the reinforced concrete forest. Cellular at the site control level is obtained from the GIS raster data conversion, whose encoding properties are given in the site design results. Meanwhile, the establishment of urban and rural space relies on the cellular growth model of venue level.

\section{STREAMLINE NETWORK LEVEL}

Although the function of the bubble chart shows the flow line connection relationship between functional areas, the initial growth location of cellular in functional areas remains to be determined. This paper sets this position at the nodes of the flow line network level, mainly streamline intersections in functional areas, and optimizes its location with adoption of inherit algorithm. GA chromosome means an entity with individual characteristics, where the most simple column grid coordinates will be built within the boundaries of functional areas, the decimal number of southwest corner of the column grid unit is 0101, the first two's direction is West to East, the latter is South to North direction. And then transform each node column grid coordinates in decimal into binary coordinates, such as the conversion of 0101 into 000 0010000010101 , which constitutes a string of 0's and 1's chain whose length is 12. All individuals at the intersections of flow lines in functional areas constitute the initial population where there are four types of nodes. The first is the intersection of the main traffic flow lines between functional areas; the second is the intersection of tandem mainstream line and tributary lines (evacuation routes) in functional areas; and the third is the streamline interchange of the inside and outside functional areas, that is the entrances and exits. The fourth is the connection nodes intra functional areas. The first two nodes are called core nodes in functional areas, where only one has core node, and the latter two are called outreach node ribbon. The third node is taken as the core node architecture with vertical boundary point, and the fourth node temporarily takes the midpoint of the two core nodes.

The information on the relationship between the core network node streamline messaging bubble chart is shown in the connection matrix $C$. Between each element a node pair represents whether have or lack a connection. Thus, according to whether there is a direct connection between the node $\mathrm{m}$ and node $\mathrm{n}$, gives 0 or 1, i.e. $C_{m n}=1$ or 0 . With the matrix $C$ and the initial population, traffic information with shortest distance between each pair of nodes streamline core network can be calculated; this information indicates the distance of matrix P. Traffic between the nodes of each element Pmn represents cellular network through which the number of columns pass.

Building space meet the individual spatial behavior of people using the building, i.e. using the human colony for construction activities forms specific actions flow line. Spatial behavior of individuals, who are building on their own social and economic use of physical characteristics (such as requirements, values, feelings, premonition) and specific behaviors as perceived by the external environmental factors generated by the interaction of these external factors with the network level, controls the factors affecting the flow line level. Individuals have impressions on the environment through the value system, then they make space action decisions.

Take Fig. (1) as an example; the building is a nursery home, which has 10 zones in total including: service area, disability conservation units and daily care areas, semideactivation conservation units and daily care areas, management offices, health regions, ambulance and hospice area, communication center, cultural and entertainment area, kitchen area, and laundry area. And each zone can be decomposed for the specific use of the room (excluding traffic space and health auxiliary space). The hospital has a total of 166 available rooms of 60 categories, which can accommodate 80 elder people, 20 for health care, 10 for the management office staff, 10 for logistics personnel, permanent endowment of this building is 120 with regular visits of 5 people, with 125 people in total. Dividing from identities, there are17 categories in total: self-care model for the elderly, elder people who lost self-care ability, dean of nursing home, office director, employees, physicians, nurses, logistics director, store clerks, cooks, kitchen staff, cleaning staff, laundry staff and visitors. Involved flow lines are listed as follows:

Registration flow line: Check in----- ---- health assessment, flat and vertical transportation ---- curing unit (disability, semi-disability area); Activity Stream Line: ---- conservation unit level, vertical transportation ---- communication hall, entertainment, multi-purpose hall, rehabilitation, counseling (choose one from multiple choices); Medical flow line: ---- conservation unit level, vertical transportation ---health regions, special infectious disease isolation; Dying flow lines: conservation unit designed Gallery ---- ---- ---ambulance ---- Hospice ---- taking out by families.

Management of office streamlines: Open office foyer ------- Check in ---- Serve at Service Area; Open office foyer --- ---- ---- Office training, jobs data; Open office foyer ---- ------- Office Services (conservation units, health care, public events, logistics); Office foyer ---- ---- administrative office punch; Office foyer punch ---- ---- ---- Social Work area of vertical transportation Counseling; Office foyer ---- ---punch training room, maintenance unit, multi-purpose hall, psychological counseling content Health care personnel flow lines: The main entrance corridor ---- ----- area of the department of health posts ----- Health district level, vertical transportation ---- conservation areas domiciliary observation unit; Health Care District ---- ---- training or multi-purpose hall or district department of health, health-related content; Health Care District ---- Check the service area to assess post Staff dining, rest flow lines: Office, healthcare, conservation 
unit, kitchen laundry room of transport ----- -------- restaurant workers; Each unit ---- levels, vertical transportation office foyer ----- ----- leave from work.

Dinner uniform associated flow lines: waiter: Kitchen, pantry, laundry room spouts ------ ------ wagons corridor conservation unit specially prepared meals twice ------ ----unit or nursing station traffic - - each bedroom. Recycling flow lines and vice versa.

Internal kitchen flow lines: Waiter flow line: hall processing ---- ---- ---- receipt pantry ----- catering, maintenance unit; Streamline kitchen staff: entrance hall ---- ---- dressing and processing jobs.

Laundry flow lines: Waiter flow line: Laundry ---- ---accept payment; Laundry man flow line: Entrance ---- laundry room

Elder visitors flow line: registration service at the entrance---- corridor ----- ----- conservation unit bedroom ----family room.

Waste handling streamline: Conservation bedroom ----- ---- wash dirty ----- laundry room or scrapped. We use the above construction and streamline information flow line for room arrangement design phase analysis. At the flat analysis phase of functional area, we need to combine and simplify it according to functional areas in order to make use of distance matrix $\mathrm{P}$ for calculation.

Define $d_{i j}, v_{i j}, f_{i j}$, as Cellular access number, the speed factor, frequency factor of the index of the $i$-net humanoid $j$ th flow line, and the mastery efficiency of architecture streamlines is:

$$
T=\sum_{i=1, j=1} d_{i j} v_{i j} f_{i j}
$$

The unit of the speed factor is $\mathrm{m} / \mathrm{s}$, the value equals to the speed of group of people $i$ pass flow rate of the $j$-th line, the frequency factor for the $i$-th class pass average number of people per day in the $j$-flow line. Set building perimeter boundaries as $L_{\max }$, Let

$$
f_{t}=\frac{T}{L_{\max } \sum_{i=1, j=1} v_{i j} f_{i j}}
$$

Computing certain functional area, the core node to node length and outreach for $s_{i}$, functional area of approximately $A$, $p=S_{i} / A$ the membership function can be expressed as:

$$
f_{c}^{i}=\left\{\begin{array}{l}
t_{1}<p<t_{2} \\
1-\frac{t_{1}-p}{t_{1}} \quad 0<p<t_{1}
\end{array}\right.
$$

Transfer the restricted area size into the penalty function involved in fitness value calculation, set the number of functional areas, and obtain the final value. $f_{c}=\frac{\sum_{i=1}^{m} f_{c}^{i}}{m}$

Set $p_{t}$ and $p_{c}$ as weight and the fitness value respectively, calculated according to the genetic algorithm to obtain satisfactory core node coordinates, which is the starting point for the growth of functional areas.

\section{CONTROL FACTORS LEVEL}

In terms of location choice of functional areas, various factors which influence its growth can be summarized in force [13]. Functional areas can only grow toward the path with the least resistance or the biggest attraction to the gravity and restriction is a result of joint influence of various factors. But under different circumstances, some ribbon Growth factors have a large space force, and some space for the growth of the force is generated by a small spatial factors.

According to the principles of physics in action force, in every functional area location of choice, the ribbon growth factors influencing the formation of the force are divided into functional areas gravitational growth, ribbon growth resistance, and ribbon growth friction. Ribbon growth role of choice depends on the behavior of the various forces and the size of all the space. Among them, the growth of gravity is favorable for constructing growth factors, and growth resistance is favorable for building growth unfavorable factors, which are formed by the external environment. Some of the control elements of the gravity are grown within the cell column grid within a certain range of their produce, such as convenient transportation, good environment, complete infrastructure, agglomeration economies etc., while some other control elements within its column grid around a range of unit generate growth resistance [14], such as a variety of sources. Friction is a function of the growth characteristics of the decision of the district itself, mainly referring to the column grid cell growth needed to reach across the threshold. As long as this threshold is crossed, it will eliminate the negative factors.

In this paper, the force is used to quantify spatial fuzzy mathematics requirements for controlling attribute information layer count quantitative processing, such as conversion of value to the corresponding level, column cellular network on different functional areas etc. According to the strength of demand of growth plan given the appropriate buffer range, plus stacking it with the control of the buffer range for controlling information layer for mass property calculations and statistics, since the control layer of raster elements, counts the number of the grid cells in a buffer zone.

Here, $F_{i}$ represents the $i$-th control layer properties of functional units calculating result information, i.e.,

$$
F_{i}=\frac{\sum_{j=1}^{m} N_{j} \times p_{j}}{m}
$$

which, $N_{j}$ is the control layer attribute values for different elements of the grid; $p_{j}$ is the area ratio for the elements of 
the grid in the buffer zone occupied by the value range; $m$ is the number of different types of elements of the grid.

Set membership function

$f_{i}=\frac{N_{\text {max }}-F_{i}}{N_{\text {max }}-N_{\text {min }}}$

where, $N_{\max }$ and $N_{\min }$ are respectively the maximum and minimum amount of calculation attribute values.

\section{COLUMN GRID UNIT RULES OF CONDUCT}

The column grid cell behavior uses the collection process denoted by the symbol:

$B_{l}^{t}=\left\{E_{l}, N_{l}, T_{l}, F_{l k}, N_{k}, T_{k}, C_{k}, K_{l}\right\}$

where,

$B_{l}^{t}$---from time $t$ to $t+1, l$ is the location column grid cell behavior, that is to maintain the cellular functional area to which it now belongs, or converted into units of another functional area it belongs, or by conversion of open space into a functional unit, or converted into an open space, which includes the following course of action in a series of actions and processes;

$E_{1}$---energy value of units is determined, it is desirable, "Youth", "middle", "elderly" a state in the middle of the three;

$N_{l}$---check ribbon around the adjacent unit cell configuration, determine the behavior of functional units in accordance with the neighboring conditions;

$T_{l}$--- traffic flow lines around the condition check at layer $l$ to determine whether the development of conditions are met;

$F_{l k}$---generate new functional unit location and function of the process type of method, $k$ represents the positional newborn functional unit;

$N_{k}$---express check newborn adjacent functional unit configuration, determine whether the survival conditions are met;

$T_{k}$---express check traffic conditions around the newborn at the functional unit $k$, determine whether the survival conditions are met;

$C_{k}$--- check the control factor represents the functional unit $\mathrm{k}$ layer at the newborn, to determine whether the survival conditions are met;

$K_{l}$---indicates at time $t$, the model for the construction of the overall growth rate of the control.

The above process can be broadly grouped into four key steps.

\section{(1) Determining the functional unit energy value}

In the construction growth model, give Ribbon Agent with vital signs and divide it into three stages of "growth", "mature", "evolution", and in terms of column grid unit, based on its location, each unit is divided into "Youth", "middle-aged "," old " stages. Thus at some time, all the column grid unit may be divided into three types corresponding to the different types of units produced along different behavior rules.

$$
B(t)=B^{i}(t)+B^{m}(t)+B^{d}(t)
$$

$B^{i}(t)$ on behalf of the youth column grid unit; its energy value is maximum. It can not only continue to exist in the next moment, but also it has a strong development capacity, that is the potential to generate new functional units at its periphery;

$B^{m}(t)$ on behalf of middle column grid unit, which has low energy value, and does not have the ability to develop and thus maintains the existing state at the following moments;

$B^{d}(t)$ on behalf of the elderly column grid unit, whose functional units are at the edge of life, and doesn't have developmental potential and is going to be perished and vacant next moment.

Defining energy value characteristics of functional units has a practical meaning. Whatever the functional units are in the new emerging period, its surroundings development is boomed.

(2) Check the condition of the definition and the adjacent situation

Neighbor is one of the most important concept regarding cellular automata mode. In building all growth models, we adopt extended Moore neighbor type, which is one of the most common types of neighbors. It is based on the Quartet for the two-dimensional mesh generation architecture plane models, such as the radius of a given neighborhood $r=1$, then the neighbor set for the previous cellular centered $3 \times 3$ squares.

In the construction growth model, any cellular behavior is affected by the state of eight neighbor surroundings. Take whether a certain comprehensive index is qualified in the neighbor community into consideration, and neglect specific space combination of unit neighbors.

(3) Produce new functional unit

When the value of a functional unit equals to youth, as well as qualifies neighbors and smooth flow lines, it can generate a new subunit with similar function of parent units as a parent unit.

(4) Supervision and control of living conditions of new functional units

After the location and type of neonatal unit are determined, we also need to go through a series of checks in order to finalize the process of birth of newborn unit. As long as any of the conditions is not met, the entire process fails. Specifically, the newborn unit also goes through check for five aspects.

(a) Check whether the newborn unit is occupied. Neonatal unit can only be born in vacancy. If that position is occupied by Ribbon unit, then the birth of the newborn unit is a declared "abortion." 
(b) Check the newborn unit based on the ribbon column grid unit level neighborhood conditions. For example, when the number of neighbors within the range of other functional units is greater than a certain threshold, then it is unreasonable to generate this new unit. The process is shown as Equation $N_{k}(7)$.

(c) Check the newborn unit at the level of neighborhood traffic situation. Neonatal unit must be near the lines of communication, such as there should be no lines of communication within the scope of the neighbors, since it is the behavior of abortion. The process is shown as Equation $N_{k}(7)$.

(d) Check on the control plane, the position of the control condition. The process is shown as Equation $N_{k}(7)$. Integrated level control factor layer is superposed through a variety of factors such as spatial analysis obtained. Controlling factors in each column grid unit level position require a survival probability of different types of functional units and a unit of flow lines. So, control factor layer behaves as a three-dimensional matrix of control probability; when the probability value is between 0.0 and 1.0 , the size of its probability value reflects the extent to which the development of such functional units is either encouraged or restricted.

(e) Check the overall control conditions. The process is shown as Equation $N_{k}(7)$. In general, control of the border of single building, forms a neat appearance. The four corners of the building preferably form a rectangle, with corners aligned.

\section{CONCLUSION}

Architectural features and style is just one kind of method for generating function modeling [15]. Some scholars have called architectural style as shaping, which reflects the different building generated ideas in the world. Individual building can be seen as a large volume of sculpture art, and the method of "subtraction" is mainly used in detailing sculpture production process. The first step is generation of enough huge architecture in planning regulations in a limited area, the overall architecture of the embryo meets the requirements of the volume rate, and then "dig subtract" until get stereotypes.

This paper proposed a relatively "addition", the shape growth required at a fixed position of the flow line case, but the result has a certain randomness. To optimize the shape, the flow line position must have partial adjustments. As developing a new area in the city, the old roads are gradually abandoned, the old community demolished, construction of new communities expand in the vicinity of the new transit. Growth model can be adjusted to optimize the flow line by using genetic algorithms, which is also one of the optimization algorithms to generate a row of rooms. Flow lines need refinement and functional areas should be further fine-tuned in the second phase of architectural generative design, which remains to conduct further detailed studies.

\section{CONFLICT OF INTEREST}

The authors confirm that this article content has no conflict of interest.

\section{ACKNOWLEDGEMENTS}

Declared none.

\section{REFERENCES}

[1] B. Li, and J.P. Qian, "Research on cellular automata architectural design generation method", Journal of New Building, vol. 3, pp. 103-108, 2009.

[2] S. Chen, Urbanization and Geographic Information System. Science Press, China: Beijing, 1999, pp. 171-197.

[3] A. Nikos, and C.Q. Li, Twelve New Architectural Theory: Urban Design Theories Based on Latest Mathematical Methods. China Building Industry Press, China: Beijing, 2014, pp. 75-90.

[4] X.J. Liu, Modern Architectural Theory. China Building Industry Press, China: Beijing, 2008, pp. 231-269.

[5] H. Zhang, "Rational design features and technical methods of the building generation", Journal of Jiangxi Building Materials, vol. 21, p. 18, 2014.

[6] Z.T. Li, Architectural Design Method. China Building Industry Press, China: Beijing, 2010, pp. 43-95.

[7] J.L. Schiff, Cellular Automata: A Discrete View of the World. Published by Wiley \& Sons, Inc. U.S.: NJ, 2011.

[8] J.J. Wu, and H. Zhao, Urban Transport System Complexity - Complex Network Method and its Application. Science Press: China, Beijing, 2010, pp. 17-62.

[9] B. Li, and D.Q. Han, "Understanding and prospects of architectural design generation techniques", Architectural Journal, vol. 13, pp. 96-100, 2011.

[10] B. Li, Z.F. Guo, and R. Li, "Digital chain building generation gap filling technology", Architectural Journal, vol. 15, pp. 20-25, 2013.

[11] H. Tian, "Study based Architectural Design Method to Generate Complex Adaptive Systems", M.S. thesis, Dalian University of Technology, Dalian, China, 2011.

[12] W.G. Xu, W.X. Huang, and M.Y. Jin, "Procedural logic - nonlinear architectural design - the technical route to explore", Journal of City Building, vol. 9, pp. 10-14, 2010.

[13] G. Lynn, Animate Form. Princeton Architectural Press, U.S.: NY 1998.

[14] G. Lynn, Folds, Bodies and Blobs. Princeton Architectural Press, U.S.: NY, 1998.

[15] W.X. Huang, and W.G. Xu, "Form-finding of nonlinear architectural design”, Architectural Journal, vol. 11, pp. 96-99, 2009.

\begin{tabular}{lcc}
\hline Received: January 25, 2015 & Revised: March 01, 2015 & Accepted: March 10, 2015 \\
(C) Hou and Xu; Licensee Bentham Open. &
\end{tabular}

This is an open access article licensed under the terms of the Creative Commons Attribution Non-Commercial License (http://creativecommons.org/licenses/by-nc/4.0/) which permits unrestricted, non-commercial use, distribution and reproduction in any medium, provided the work is properly cited. 